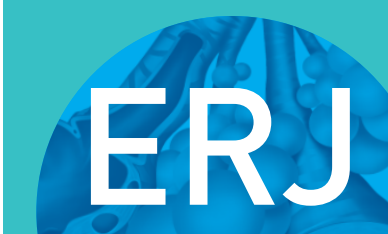

open research

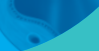

\title{
Reducing the frequency of respiratory tract infections in severe neurological disorders by inhaled antibiotics: a retrospective data analysis
}

\author{
Maximilian Eckerland (D), Claudia Bock, Margarete Olivier, Leopold Pichlmaier (1), \\ Mathis Steindor and Florian Stehling
}

Affiliation: Dept of Pediatric Pulmonology and Sleep Medicine, University of Duisburg-Essen, Children's Hospital, Essen, Germany.

Correspondence: Maximilian Eckerland, Dept of Pediatric Pulmonology and Sleep Medicine, University of Duisburg-Essen, Children's Hospital, Hufelandstrasse 55, 45122 Essen, Germany.

E-mail: maximilian.eckerlandauk-essen.de

\section{ABSTRACT}

Background: In patients with severe neurological impairment, recurrent respiratory tract infections frequently occur as a result of impaired clearance of airway secretions and microbial airway colonisation. We hypothesised that inhaled antibiotic therapy may improve the morbidity of these patients.

Methods: A retrospective data analysis of 20 patients (11 nontracheotomised and nine tracheotomised) with neurological impairment and microbial airway colonisation was carried out at a children's university hospital. Two questionnaires that asked about the number of respiratory tract infections, antibiotic therapies and hospitalisations were distributed to the patients/caregivers: a first questionnaire representing the 12 months prior to the initiation of inhaled antibiotics and a second questionnaire describing the first 12 months under therapy.

Results: During the first 12 months of therapy, the frequency of respiratory tract infections among all participants was reduced from a mean of 6.8 episodes (median (interquartile range (IQR)) 6.0 (4.0-10.0) episodes) to a mean of 2.5 episodes (median (IQR) 2.0 (1.0-3.0) episodes; $\mathrm{p}<0.001$ ). In addition, a significant decrease of systemic antibiotic therapies (mean 7.7, median (IQR) 6.0 (4.0-10.0) versus 2.5, 2.5 $(0.0-3.75)$ episodes; $\mathrm{p}<0.001)$ and hospitalisations (mean 3.9, median (IQR) $3.5(1.0-5.0)$ versus $0.9,0.0$ $(0.0-1.0)$ episodes; $\mathrm{p}<0.001)$ was noted. This significant therapeutic effect could be demonstrated in a subgroup analysis in both tracheotomised and nontracheotomised subjects. The reduction of respiratory tract infections and systemic antibiotic therapies (and thus the therapeutic success) was significantly greater in the nontracheotomised group compared with the tracheotomised group.

Conclusions: The presented data suggest that inhaled antibiotics might play a role in treating recurrent respiratory tract infections in neuromuscular diseases.

@ERSpublications

Inhaled antibiotic therapy offers a novel approach to the prevention of respiratory tract infections in patients with severe neurological impairment who have chronic microbial airway colonisation and recurrent respiratory tract infections http://bit.ly/2HQRyz0

Cite this article as: Eckerland M, Bock C, Olivier M, et al. Reducing the frequency of respiratory tract infections in severe neurological disorders by inhaled antibiotics: a retrospective data analysis. ERJ Open Res 2019; 5: 00149-2018 [https://doi.org/10.1183/23120541.00149-2018].

Received: Sept 062018 | Accepted after revision: May 142019

Copyright $\odot E R S$ 2019. This article is open access and distributed under the terms of the Creative Commons Attribution Non-Commercial Licence 4.0. 


\section{Introduction}

Patients with severe neurological impairment such as neuromuscular disorder (NMD) or infantile cerebral palsy (ICP) regularly suffer from respiratory complications such as atelectasis or respiratory tract infections $[1,2]$. Primarily, the lung itself is considered to be healthy and all respiratory morbidity is believed to be secondary due to chest wall restriction and expiratory muscles weakness, resulting in cough insufficiency and ineffective airway clearance [3]. Continuous retention of airway secretions might increase the risk of microbial airway colonisation with potential pathogenic bacteria. Studies have shown that bacterial colonisation of the upper airways with Gram-negative bacteria or Staphylococcus aureus occurs in $~ 50 \%$ of NMD patients regardless of a respiratory tract infection [4]. Furthermore, patients with ICP may develop abnormal bacterial airway flora due to continuous (micro-)aspirations as a result of bulbar dysfunction or gastro-oesophageal reflux [5-7]. The presence of a tracheostomy further raises the probability of persistent bacterial colonisation of the lower respiratory tract in patients with NMD or ICP [8,9]. Pseudomonas aeruginosa is the most commonly detected pathogen in tracheotomised patients $[10,11]$ and data suggest that $P$. aeruginosa also plays a role in nontracheotomised children with neurological impairment $[5,7,12]$. As a result of chronic bacterial colonisation, airway inflammation and recurrent respiratory tract infections, particularly pneumonia, may evolve, and these are the most common causes of hospitalisation in both tracheotomised [13] and nontracheotomised [14] patients with neurological impairment. These recurrent exacerbations aggravate the respiratory situation $[15,16]$ and eventually lead to respiratory failure, as increased mucus retention further impairs limited pulmonary gas exchange.

Morbidity and mortality of patients with neurological impairment and respiratory complications, particularly in NMD patients, have been reduced significantly since structured airway management including noninvasive ventilation and mechanically assisted cough techniques became standard treatment [3, 17]. Mechanically assisted cough supports chest physiotherapy and primarily induces mechanical secretolysis. However, there are currently no established therapeutic concepts to manage bacterial airway colonisation in these patients. The inhalation of antibiotics is therefore a promising therapeutic approach. Benefits include high local drug concentration, low systemic toxicity, feasibility in an outpatient setting and easy long-term use without mandatory permanent medical monitoring. Since the 1990s, antibiotic inhalation in patients with cystic fibrosis (CF) has been of fundamental importance for the eradication therapy of an early or intermittent colonisation [18] and the suppression therapy of a chronic infection [19] with P. aeruginosa, resulting in a significant improvement of lung function and a decrease in hospitalisations due to an acute exacerbation $[20,21]$. In the meantime, the use of inhaled antibiotics has been extended to treat non-CF bronchiectasis [22], ventilator-associated pneumonia [23] and nontuberculous mycobacterial infections [24]. Recently, two small case series also suggested a benefit of inhaled antibiotics in patients with neurological impairment and frequent respiratory exacerbations [25, 26], but larger studies are missing.

We hypothesised that reducing the bacterial load in patients with severe neurological impairment by inhaled antibiotics has an impact on the frequency of respiratory tract infections, the need for systemic antibiotic therapy and the number of hospitalisations.

\section{Methods}

Data collection

This data analysis was carried out at the Dept of Pediatric Pulmonology and Sleep Medicine of the University Hospital Essen (Essen, Germany). All patients or their caregivers were asked to fill out two questionnaires (with identical questions): a first questionnaire representing the 12 months prior to the initiation of therapy with inhaled antibiotics and a second questionnaire describing the first 12 months after the start of therapy. We asked for 1) number of respiratory tract infections, 2) number of systemic antibiotic therapies, 3) number of hospitalisations, 4) use of noninvasive ventilation and 5) use of mechanically assisted cough techniques. Results of the microbiological samples (throat swab/tracheal secretions) collected during the outpatient and inpatient visits were collected from the patient's charts.

Inhaled antibiotic therapy was started between 2011 and 2015. The indication was based on clinical aspects (high number of respiratory tract infections, chronic bacterial airway colonisation and colonisation with $P$. aeruginosa). Data collection was carried out in 2016. 20 subjects (14 females and six males) with neurological impairment and recurrent respiratory tract infections were investigated.

The Ethics Committee of the University of Duisburg-Essen (Essen, Germany) approved this data analysis (internal study 16-7032-BO). Informed consent was obtained from all study participants or their legal guardians. None of the eligible participants declined to participate in the study.

\section{Statistical analysis}

Statistical analysis was performed using SPSS version 24 (IBM, Armonk, NY, USA). The normal distribution of our data was tested using the Shapiro-Wilk test. As most of the data failed to reach normal 
distribution and the sample size of 20 subjects was small, further data analysis was carried out with nonparametric tests: the Wilcoxon signed-rank test was employed for the comparison of results prior to and during therapy with inhaled antibiotics, and the Kruskal-Wallis test was employed for subgroup analysis of tracheotomised and nontracheotomised subjects. A p-value $<0.05$ was considered statistically significant. Mean, median and interquartile range (IQR) were used for descriptive data analysis. The graphs were created using Prism version 7.04 (GraphPad, San Diego, CA, USA).

\section{Results}

Subjects

Subject characteristics are summarised in table 1 . Mean \pm SD age was $12.1 \pm 10.1$ years (range 1-37 years). Eight subjects suffered from ICP, four from congenital myopathy, three from spinal muscular atrophy, two from Duchenne muscular dystrophy, two from neuronal ceroid lipofuscinosis and one from tracheomalacia (as part of VACTERL (vertebral defects, anal atresia, cardiac defects, tracheo-oesophageal fistula, renal anomalies and limb abnormalities) association). Nine subjects had a tracheostomy (45\%) and invasive ventilation therapy, while noninvasive ventilation as a consequence of respiratory insufficiency was used by five subjects (25\%). All home mechanical ventilation therapies were initiated prior to study entry. Accordingly, none of the patients started mechanical ventilation during the study period.

15 subjects (75\%) used mechanically assisted cough during the whole study period. In four of these subjects mechanically assisted cough was started simultaneously with or shortly after the onset of inhaled antibiotic therapy. The remaining 11 subjects continued the mechanically assisted cough therapy, which started $>1$ year prior to the initiation of inhaled antibiotics.

Inhaled antibiotic therapy was performed in 15 subjects (75\%) with tobramycin (80 mg twice daily) and in five subjects (25\%) with colistin $\left(1 \times 10^{6} \mathrm{IU}\right.$ twice daily), each continuously for at least 1 year of therapy.

\section{Morbidity prior to and during inhaled antibiotic therapy}

In the 12 months prior to initiation of inhaled antibiotic therapy virtually all subjects exhibited colonisation with potential pathogenic bacteria, of which $P$. aeruginosa occurred most frequently in 16 subjects (80\%). After the first 12 months of inhaled antibiotics, $P$. aeruginosa was still cultured in 10 out of the 16 subjects (62.5\%) in whom P. aeruginosa was previously detected.

\section{TABLE 1 Subject characteristics}

$\begin{array}{lc}\text { Underlying disease } & \\ \text { Infantile cerebral palsy } & 8 / 20(40) \\ \text { Congenital myopathy } & 4 / 20(20) \\ \text { Spinal muscular atrophy } & 3 / 20(15) \\ \text { Duchenne muscular dystrophy } & 2 / 20(10) \\ \text { Neuronal ceroid lipofuscinosis } & 2 / 20(10) \\ \text { Tracheomalacia } & 1 / 20(5) \\ \text { Sex } & \\ \text { Female } & 14 / 20(70) \\ \text { Male } & 6 / 20(30) \\ \text { Age years } & 12.1 \pm 10.1(1-37) \\ \text { Tracheostomy } & 9 / 20(45) \\ \text { Infantile cerebral palsy } & 3 / 8(37.5) \\ \text { Congenital myopathy } & 3 / 4(75) \\ \text { Spinal muscular atrophy } & 1 / 3(33) \\ \text { Duchenne muscular dystrophy } & 2 / 2(100) \\ \text { Noninvasive ventilation } & 5 / 20(25) \\ \text { Infantile cerebral palsy } & 2 / 8(25) \\ \text { Spinal muscular atrophy } & 2 / 3(66) \\ \text { Tracheomalacia } & 1 / 1(100) \\ \text { Mechanically assisted cough } & 15 / 20(75) \\ \text { Infantile cerebral palsy } & 4 / 8(50) \\ \text { Congenital myopathy } & 4 / 4(100) \\ \text { Spinal muscular atrophy } & 3 / 3(100) \\ \text { Duchenne muscular dystrophy } & 2 / 2(100) \\ \text { Neuronal ceroid lipofuscinosis } & 2 / 2(100)\end{array}$

Data are presented as $\mathrm{n} / \mathrm{N}(\%)$ or mean \pm SD (range). 
TABLE 2 Bacterial detection in all subjects in the 12 months prior to inhaled antibiotics and during the first 12 months of inhaled antibiotics

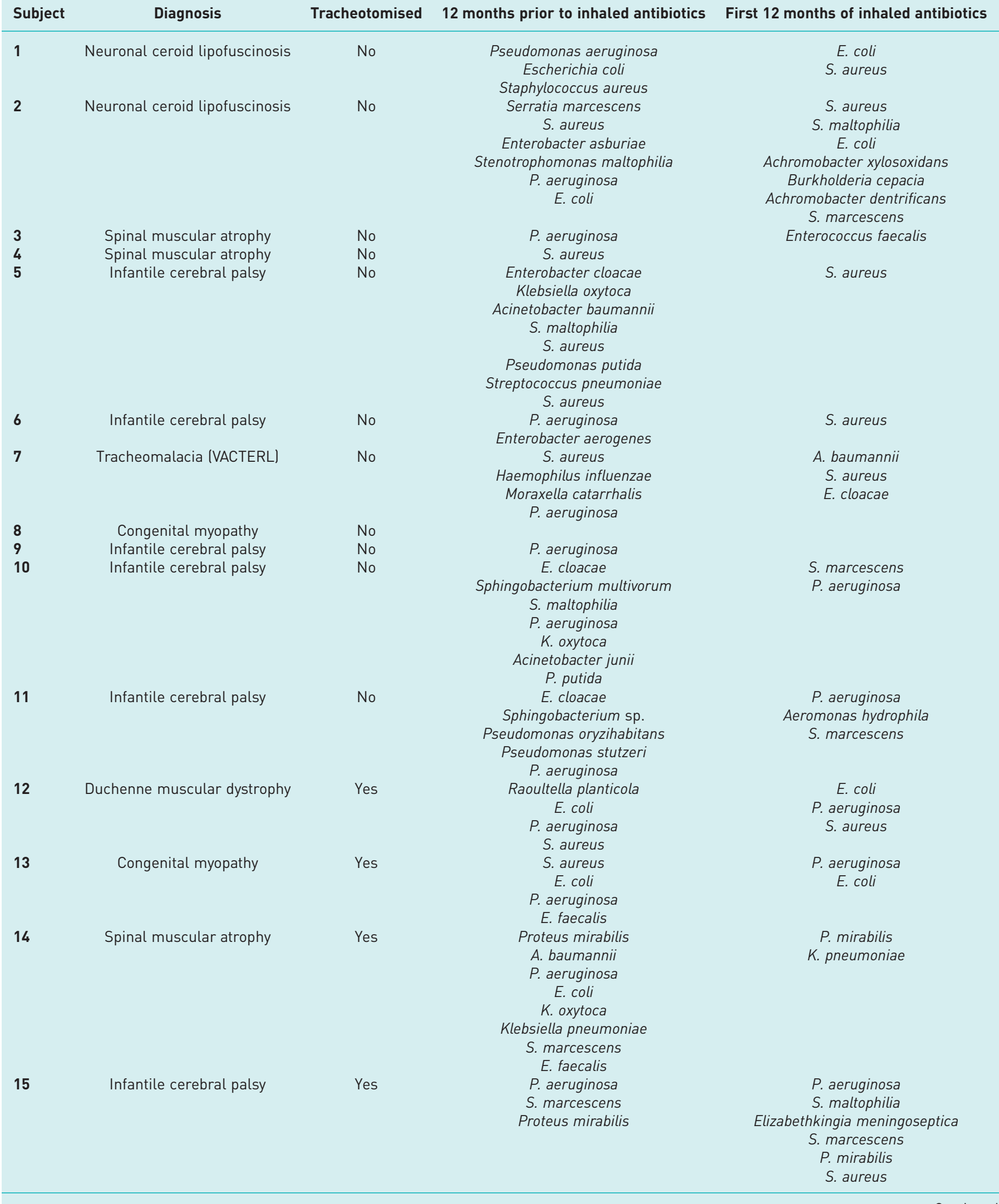


TABLE 2 Continued

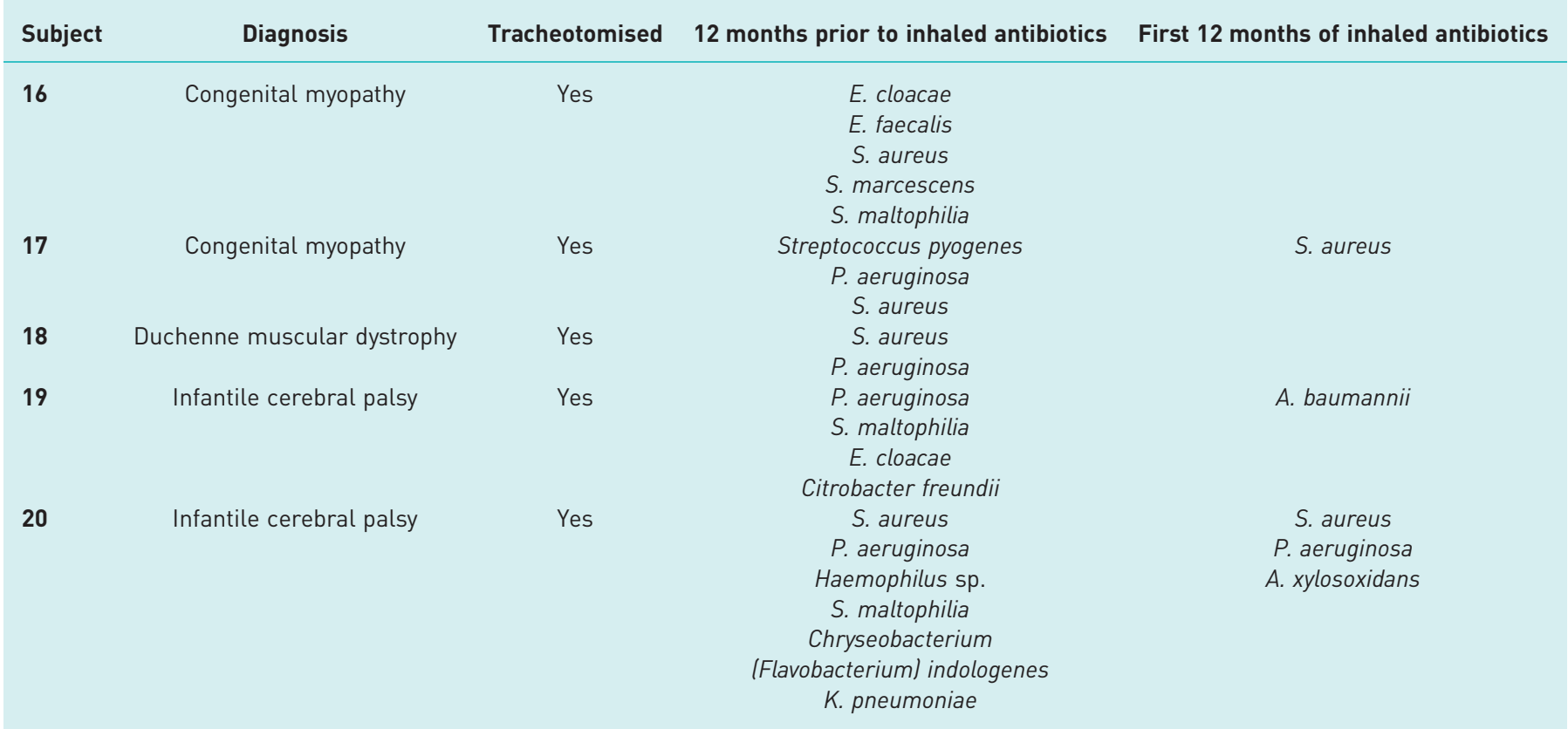

VACTERL: vertebral defects, anal atresia, cardiac defects, tracheo-oesophageal fistula, renal anomalies and limb abnormalities.

In the tracheotomised subgroup, $P$. aeruginosa was detected at least once in all but one subject. In 15 of the subjects (75\%), multiple potentially pathogenic organisms were identified. Besides $P$. aeruginosa, the most common bacteria identified were S. aureus and Stenotrophomonas maltophilia. One of the subjects started inhaled antibiotic therapy based on a history of recurrent pneumonia without detection of bacterial airway colonisation. All bacteria detected in the 12 months prior to inhaled antibiotic therapy and during the first 12 months of therapy are summarised in table 2 .

In the 12 months prior to initiation of inhaled antibiotic therapy, the subjects had a mean of 6.8 respiratory tract infections (median (IQR) 6.0 (4.0-10.0) respiratory tract infections). The frequency was reduced to a mean of 2.5 episodes in the first 12 months under inhaled antibiotic therapy (median (IQR) $2.0(1.0-3.0)$ episodes; $\mathrm{p}<0.001$ ) (figure 1). This reduction of respiratory tract infections was accompanied by a significant decrease of systemic antibiotic therapies (mean 7.7, median (IQR) 6.0 (4.0-10.0) versus 2.5, $2.5(0.0-3.75)$ episodes; $\mathrm{p}<0.001$ ) (figure 1) and number of hospitalisations (mean 3.9, median (IQR) 3.5 $(1.0-5.0)$ versus $0.9,0.0(0.0-1.0)$ episodes; $\mathrm{p}<0.001)$ (figure 1$)$. In summary, each of the 20 subjects showed significant clinical improvement with fewer respiratory tract infections, fewer antibiotic therapies and fewer hospitalisations (except for one subject who had a single hospital stay after therapy and none before treatment). The before-after graphs of each subject in terms of number of respiratory tract infections, antibiotic therapies and hospitalisations are shown in figures 2-4.

\section{Subgroup analysis: nontracheotomised versus tracheotomised subjects}

In the next step a subgroup analysis of tracheotomised and nontracheotomised subjects was performed.

\section{Nontracheotomised subjects}

In the 12 months prior to initiation of inhaled antibiotic therapy, the nontracheotomised subjects suffered from a mean of 8.8 respiratory tract infections (median (IQR) 9.0 (6.0-10.0) respiratory tract infections). In the first 12 months after inhaled antibiotic therapy had been started, we found a decrease to a mean of 3.5 episodes (median (IQR) $3.0(2.0-5.0)$ episodes; $\mathrm{p}=0.001$ ) (figure 5). Furthermore we observed a significant reduction of systemic antibiotic therapies (mean 10.3, median (IQR) 9.0 (6.0-16.0) versus 3.4, 3.0 (2.0-5.0) episodes; $\mathrm{p}=0.001$ ) (figure 6) and hospitalisations (mean 5.1, median (IQR) 5.0 (3.0-6.0) versus $1.1,1.0(0.0-2.0)$ episodes; $\mathrm{p}=0.004)$ (figure 7 ).

\section{Tracheotomised subjects}

In the subgroup of tracheotomised subjects, we also found a significant decrease of respiratory tract infections (mean 4.3, median (IQR) 4.0 (3.5-5.0) versus 1.1, $1.0(0.0-2.0)$ episodes; $\mathrm{p}=0.008$ ) (figure 5), 


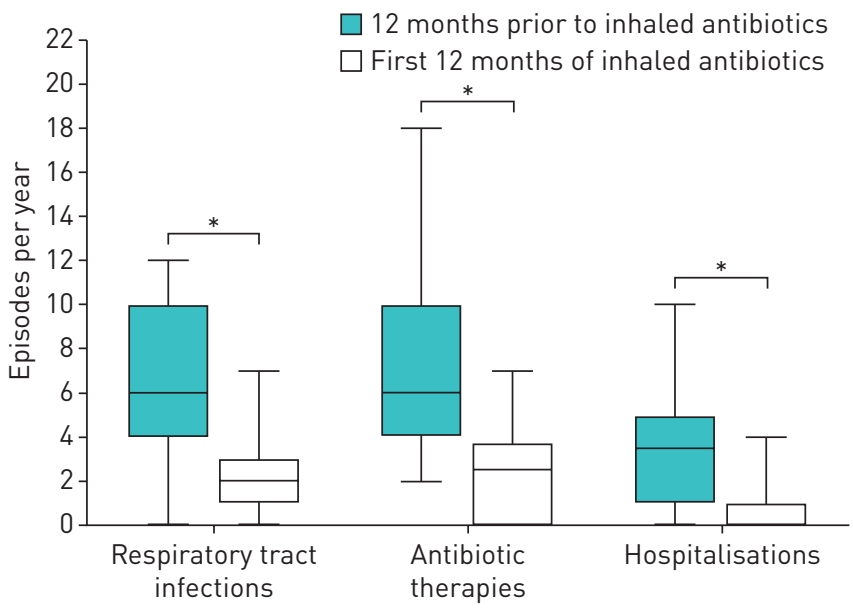

FIGURE 1 Box plots of the frequency of respiratory tract infections, antibiotic therapies and hospitalisations in the 12 months prior to inhaled antibiotic therapy and during the first 12 months of inhaled antibiotic therapy. Data are presented as median with inter-quartile range (boxes) and range (whiskers). *: $p<0.05$ (statistically significant).

systemic antibiotic therapies (mean 4.6, median (IQR) 4.0 (3.0-5.5) versus 1.4, 0.0 (0.0-2.5) episodes; $\mathrm{p}=0.016$ ) (figure 6) and hospitalisations (mean 2.3, median (IQR) $1.0(1.0-3.5)$ versus $0.6,0.0(0.0-0.5)$ episodes; $\mathrm{p}=0.016$ ) (figure 7) in the first 12 months of inhaled antibiotic therapy compared with the 12 months prior to inhaled antibiotic therapy.

Success of inhaled antibiotic therapy

The absolute change in 1) frequency of respiratory tract infections, 2) number of antibiotic therapies and 3) number of hospitalisations between the two periods can be considered as therapeutic success. According to these results, the nontracheotomised subgroup had a significantly higher treatment effect when looking at the number of respiratory tract infections (mean 5.3, median (IQR) 5.0 (4.0-6.0) versus 3.2, 3.0 (1.5-4.0) episodes; $\mathrm{p}=0.022$ ) and antibiotic therapies (mean 6.9, median (IQR) 6.0 (4.0-10.0) versus 3.1, $3.0(1.0-4.0)$ episodes; $\mathrm{p}=0.015)$. Despite a tendency to improvement, the change in hospital admissions was not significantly different between the group of nontracheotomised and tracheotomised subjects (mean 4.0, median (IQR) 4.0 (1.0-6.0) versus 1.8, $1.0(0.5-3.0)$ episodes; $\mathrm{p}=0.067)$.

\section{Discussion}

The retrospective data analysis presented here shows that inhaled antibiotic therapy is able to improve respiratory morbidity in patients with severe neurological impairment. A significant reduction of the frequency of respiratory tract infections, systemic antibiotic therapies and hospitalisations was noticed in

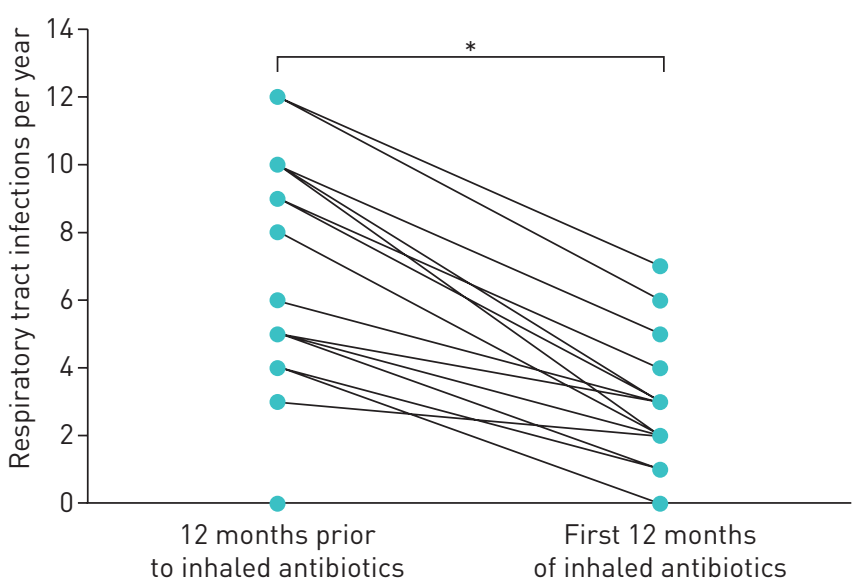

FIGURE 2 Frequency of respiratory tract infections in the 12 months prior to inhaled antibiotic therapy and during the first 12 months of inhaled antibiotic therapy. Data are presented as before-after plots for each subject. *: $p<0.05$ (statistically significant). 


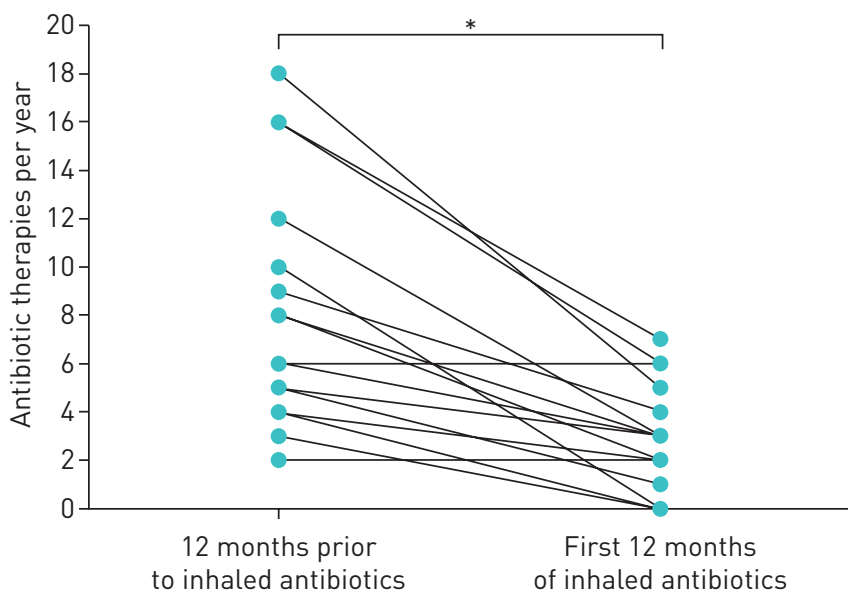

FIGURE 3 Frequency of antibiotic therapies in the 12 months prior to inhaled antibiotic therapy and during the first 12 months of inhaled antibiotic therapy. Data are presented as before-after plots for each subject. *: $p<0.05$ (statistically significant).

the entire study population as well as for the subgroup of tracheotomised and nontracheotomised subjects. This is the first study systematically describing the impact of inhaled antibiotics in this patient group.

People suffering from neurological disorders have a primary healthy lung, but respiratory diseases mainly determine morbidity and mortality. Effective management of hypoventilation and airway secretions has led to a substantial improvement of their prognosis [27-29]. However, the main causes of hospitalisation continue to be respiratory tract infections $[13,14]$, which are aggravated by permanently impaired clearance of airway secretions and associated bacterial colonisation. Likewise, long-term tracheostomy results in colonisation with $P$. aeruginosa, neutrophil airway inflammation and recurrent respiratory tract infections $[30,31]$. Although the relationship is not fully understood, data suggest that the early colonisation with $P$. aeruginosa after tracheostomy is associated with higher morbidity [31]. Chronic colonisation with Gram-negative bacteria, in particular with $P$. aeruginosa, also appears to have a significant impact on the number and duration of hospitalisations [32]. This sequence of recurrent respiratory tract infections in the context of bacterial airway colonisation due to impaired airway secretions clearance and cough insufficiency in neurologically impaired patients has already been described as secondary lung disease [26, 33]. With the increasing evidence that microbial airway colonisation and infection might play a role for morbidity, a simple therapeutic strategy is the suppression of bacterial airway colonisation with inhaled antibiotics.

Colonisation and infection with bacteria are best characterised in patients with CF, where airway colonisation and pulmonary infection due to $P$. aeruginosa are associated with neutrophil airway inflammation [34], progressive worsening of lung function [35], and increased morbidity and mortality [36].

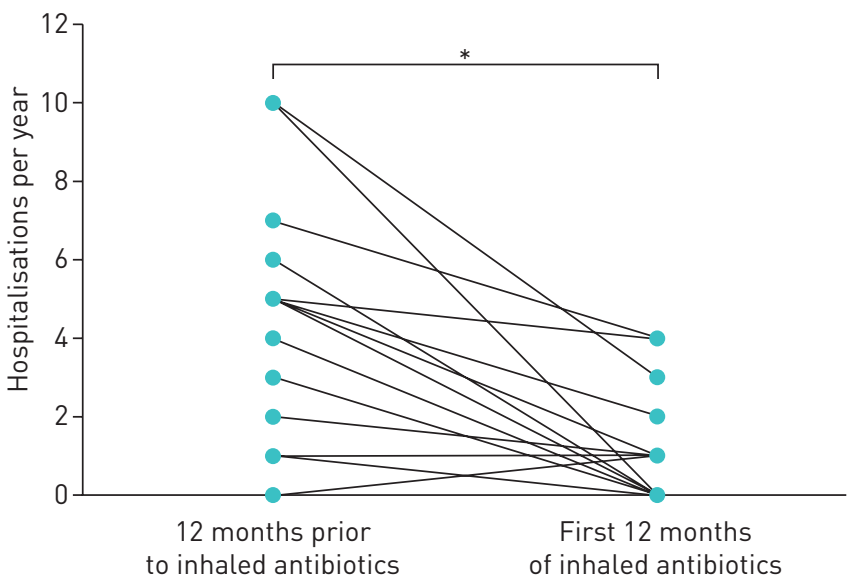

FIGURE 4 Frequency of hospitali-sations in the 12 months prior to inhaled antibiotic therapy and during the first 12 months of inhaled antibiotic therapy. Data are presented as before-after plots for each subject. *: $p<0.05$ (statistically significant). 


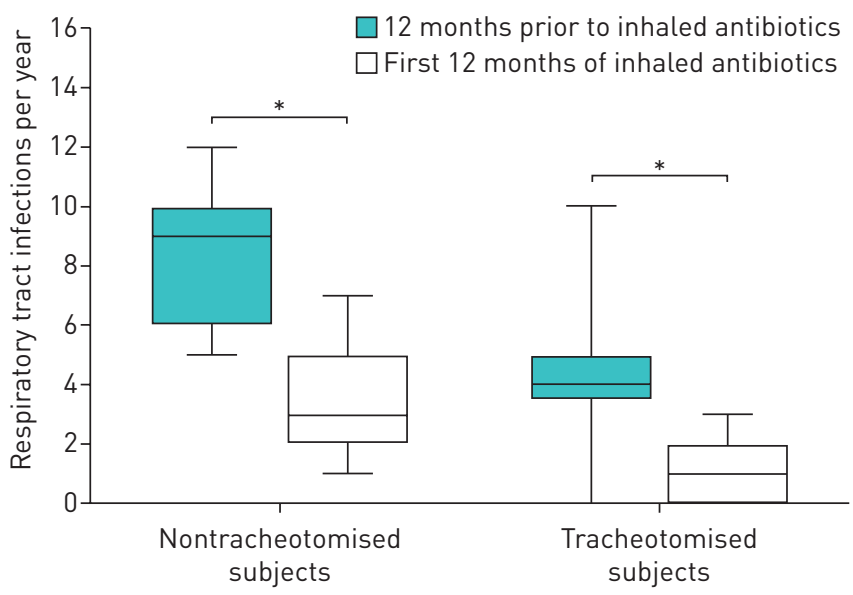

FIGURE 5 Box plots of the frequency of respiratory tract infections of the nontracheotomised subjects and the tracheotomised subjects in the 12 months prior to inhaled antibiotic therapy and during the first 12 months of inhaled antibiotic therapy. Data are presented as median with interquartile range (boxes) and range (whiskers). *: p<0.05 (statistically significant).

Permanent suppression of $P$. aeruginosa with inhaled antibiotics is the standard of care [37], and leads to an improvement of lung function and a reduction of pulmonary exacerbations [20, 21].

Evidence for the use of inhaled antibiotics in diseases other than CF is weaker, but it is becoming increasingly important, in particular in the treatment of non-CF bronchiectasis [38].

Our results are supported by two smaller reports. PLIOPLYS and KASNICKA [25] presented a case series of three nontracheotomised patients who all suffered from ICP and recurrent lower respiratory infections (3-9 episodes per year). After initiation of inhaled antibiotic therapy, the incidence of pneumonias was reduced from 19 to 10 episodes. In addition, none of the patients was hospitalised (instead of 11 episodes in the year prior to therapy). CRescimanno and Marrone [26] reported 15 NMD adults treated with inhaled colomycin $\left(1 \times 10^{6} \mathrm{IU}\right.$ twice daily) due to airway colonisation by Gram-negative bacteria and clinical signs of infection. In case of eradication, defined as three negative microbial respiratory cultures in consecutive months (an approach similar to that in CF [39]), the authors stopped the inhaled antibiotic therapy. In addition to a significant reduction in hospitalisations (mean 1.0 versus 0.0 per year; $\mathrm{p}<0.0001$ ) and pulmonary exacerbations (mean 4.0 versus 1.0 per year; $\mathrm{p}<0.0001$ ), a reduction in the need for mechanically assisted cough was achieved (mean 10.0 versus 3.0 times daily; $p<0.0001$ ). Bacterial eradication was successful in one-third of the cases. In all other subjects, a decrease of the bacterial load was observed. In our study $P$. aeruginosa could no longer be detected in 10 subjects after the first 12 months of therapy.

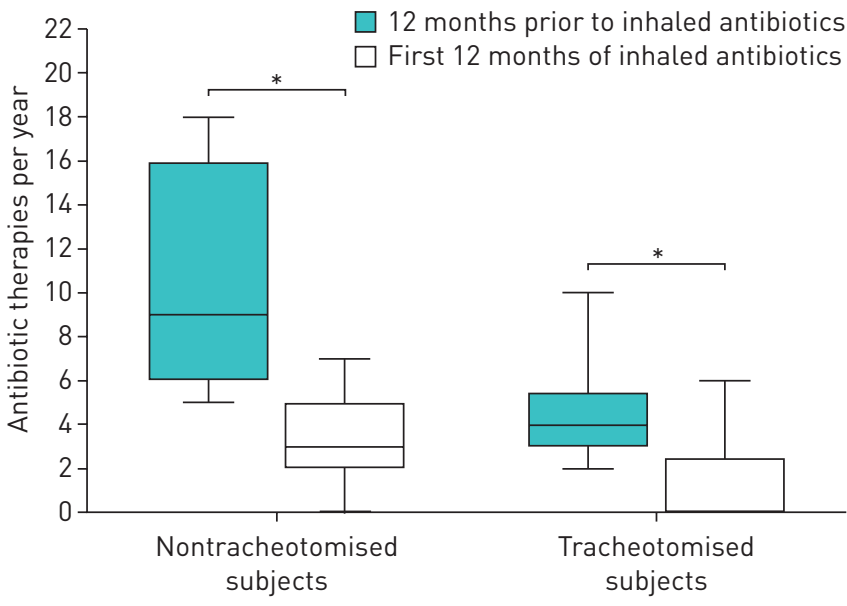

FIGURE 6 Box plots of the frequency of antibiotic therapies of the nontracheotomised subjects and the tracheotomised subjects in the 12 months prior to inhaled antibiotic therapy and during the first 12 months of inhaled antibiotic therapy. Data are presented as median with interquartile range (boxes) and range (whiskers). *: p<0.05 (statistically significant). 


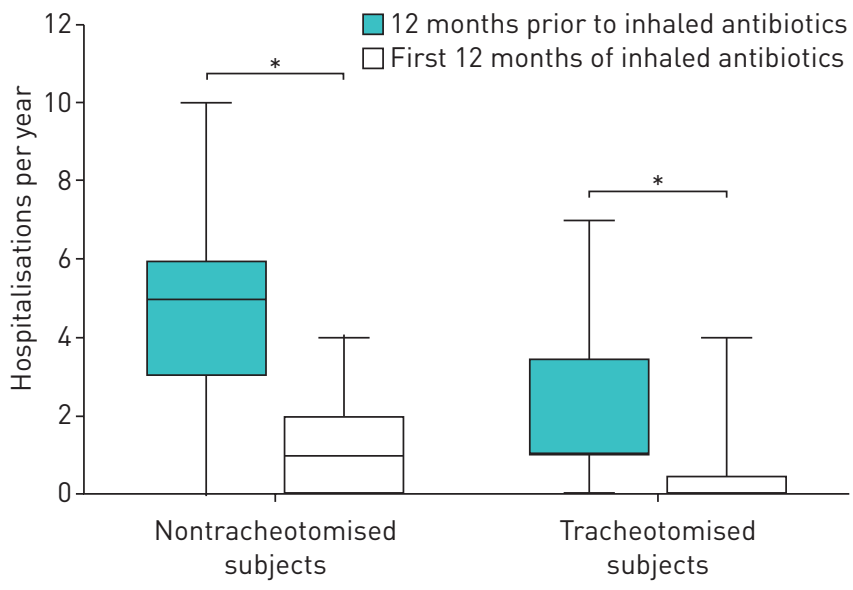

FIGURE 7 Box plots of the frequency of hospitalisations of the nontracheotomised subjects and the tracheotomised subjects in the 12 months prior to inhaled antibiotic therapy and during the first 12 months of inhaled antibiotic therapy. Data are presented as median with interquartile range (boxes) and range (whiskers). *: $p<0.05$ (statistically significant).

Both reports concluded that inhaled antibiotic therapy leads to an improvement in quality of life and lower healthcare costs, as many hospitalisations can be avoided [25, 26]. Side-effects of inhaled antibiotic therapy were not reported in these previously published studies. In the presented data analysis no systemic screening for side-effects was done. However, no obvious side-effects of antibiotic inhalation have been reported by the patients or their parents. Derived from patients with CF, it is known that inhaled antibiotics are generally safe with low toxicity and seem to be well tolerated [21]. Inhaled antibiotics therefore belong to the standard of care for patients with CF, and are applied according to national and international guidelines. It is a subject of discussion whether drug monitoring should be performed as part of inhaled antibiotic therapy [40]. So far it is not routine in CF care.

According to our data, the nontracheotomised subjects experienced a more distinct therapeutic effect compared with the tracheotomised subjects as they achieved a more pronounced reduction of respiratory tract infections, systemic antibiotics and hospitalisations during the first 12 months of inhaled antibiotic treatment. The nontracheotomised group started from a situation with significantly more respiratory tract infections, antibiotic therapies and hospitalisations prior to beginning inhaled antibiotic treatment, which offers higher potential for clinical improvement.

In addition, the indication for inhaled antibiotic therapy in tracheotomised patients may have been less precise than in nontracheotomised patients, as potential pathogenic bacteria often colonise tracheal cannulae without being clearly associated with clinical symptoms, which may explain the less complicated clinical course in tracheotomised patients.

The optimal choice of the inhaled antibiotic and its dosage remains unknown, even in the group of CF patients [18]. Plioplys and KasNicka [25] used tobramycin at a dose of $300 \mathrm{mg}$ twice daily in repeated cycles of 28 days on drug followed by 28 days off drug. The second previously published case series by Crescimanno and Marrone [26] used colomycin (colistin) as inhaled antibiotic. In the presented cohort, the lower dose of tobramycin $80 \mathrm{mg}$ inhaled twice daily (without 28 days off) was used, which might be the less aggressive and more cost-saving mode. Granted, due to the lack of evidence we often rely on experience and individual therapy response. Therefore, in some patients, we occasionally used colistin instead of tobramycin.

Some considerations should be taken into account about the present findings. Although all study participants were neurologically impaired, the study group was quite inhomogeneous. However, all subjects suffered from underlying diseases not including a primary lung disease. The indication for treatment with inhaled antibiotics was not clearly defined, but was made on clinical decisions, mainly due to a high frequency of respiratory tract infections with a documented colonisation with potentially pathogenic bacteria. However, future studies must aim to find clear and consistent treatment indications,

The analysis presented results from a retrospective study, where datasets are partially incomplete. In some cases, the questionnaires were handed out several years after the start of the therapy, which may have led to a risk of recall bias. In addition, due to the retrospective study design, objective data are missing to verify the answers to the questionnaires. Other treatments may also have influenced the study results. 
It must be emphasised that the present data analysis should serve as a first observation to demonstrate a therapeutic effect. Larger studies with a prospective randomised design are required to confirm the presented results. Finally, it should be noted that antibiotic inhalation, regardless of the results of upcoming studies, cannot replace the established therapies of mechanical mobilisation of airway secretions in patients with neurological disorders and associated lung disease.

In summary, despite the natural methodological weaknesses of a retrospective survey, our data indicate that inhaled antibiotics might provide a new therapeutic element in patients with neurological impairment that can improve the patient's morbidity, prognosis and quality of life.

Conflict of interest: None declared.

\section{References}

1 Panitch HB. Respiratory implications of pediatric neuromuscular disease. Respir Care 2017; 62: 826-848.

2 Garuti G, Verucchi E, Fanelli I, et al. Management of bronchial secretions with Free Aspire in children with cerebral palsy: impact on clinical outcomes and healthcare resources. Ital J Pediatr 2016; $42: 7$.

3 Finder JD, Birnkrant D, Carl J, et al. Respiratory care of the patient with Duchenne muscular dystrophy: ATS consensus statement. Am J Respir Crit Care Med 2004; 170: 456-465.

4 Stehling F, Pieper N, Bouikidis A, et al. Upper airway microbial colonization in patients with neuromuscular disorders. Respirology 2016; 21: 1285-1291.

5 Gerdung CA, Tsang A, Yasseen AS 3rd, et al., Association between chronic aspiration and chronic airway infection with Pseudomonas aeruginosa and other Gram-negative bacteria in children with cerebral palsy. Lung 2016; 194: 307-314.

6 Erasmus CE, van Hulst K, Rotteveel JJ, et al. Clinical practice: swallowing problems in cerebral palsy. Eur J Pediatr 2012; 171: 409-414

7 Thorburn K, Jardine M, Taylor N, et al. Antibiotic-resistant bacteria and infection in children with cerebral palsy requiring mechanical ventilation. Pediatr Crit Care Med 2009; 10: 222-226.

8 Griese M, Felber J, Reiter K, et al. Airway inflammation in children with tracheostomy. Pediatr Pulmonol 2004; 37 356-361.

9 Morar P, Singh V, Jones AS, et al. Impact of tracheotomy on colonization and infection of lower airways in children requiring long-term ventilation: a prospective observational cohort study. Chest 1998; 113: 77-85.

10 McCaleb R, Warren RH, Willis D, et al. Description of respiratory microbiology of children with long-term tracheostomies. Respir Care 2016; 61: 447-452.

11 Morar P, Singh V, Makura Z, et al. Oropharyngeal carriage and lower airway colonisation/infection in 45 tracheotomised children. Thorax 2002; 57: 1015-1020.

12 Ashkenazi-Hoffnung L, Ari A, Bilavsky E, et al. Pseudomonas aeruginosa identified as a key pathogen in hospitalised children with aspiration pneumonia and a high aspiration risk. Acta Paediatr 2016; 105: e588-e592.

13 Zhu H, Das P, Roberson DW, et al. Hospitalizations in children with preexisting tracheostomy: a national perspective. Laryngoscope 2015; 125: 462-468.

14 Dohna-Schwake C, Ragette R, Teschler H, et al. Predictors of severe chest infections in pediatric neuromuscular disorders. Neuromuscul Disord 2006; 16: 325-328.

15 Poponick JM, Jacobs I, Supinski G, et al. Effect of upper respiratory tract infection in patients with neuromuscular disease. Am J Respir Crit Care Med 1997; 156: 659-664.

16 Mier-Jedrzejowicz A, Brophy C, Green M. Respiratory muscle weakness during upper respiratory tract infections. Am Rev Respir Dis 1988; 138: 5-7.

17 Chatwin M, Toussaint M, Goncalves MR, et al. Airway clearance techniques in neuromuscular disorders: a state of the art review. Respir Med 2018; 136: 98-110.

18 Langton Hewer SC, Smyth AR. Antibiotic strategies for eradicating Pseudomonas aeruginosa in people with cystic fibrosis. Cochrane Database Syst Rev 2017; 4: 004197.

19 Ryan G, Singh M, Dwan K. Inhaled antibiotics for long-term therapy in cystic fibrosis. Cochrane Database Syst Rev 2011; 3: 001021

20 Ramsey BW, Dorkin HL, Eisenberg JD, et al. Efficacy of aerosolized tobramycin in patients with cystic fibrosis. N Engl J Med 1993; 328: 1740-1746.

21 Ramsey BW, Pepe MS, Quan JM, et al. Intermittent administration of inhaled tobramycin in patients with cystic fibrosis. Cystic Fibrosis Inhaled Tobramycin Study Group. N Engl J Med 1999; 340: 23-30.

22 Brodt AM, Stovold E, Zhang L. Inhaled antibiotics for stable non-cystic fibrosis bronchiectasis: a systematic review. Eur Respir J 2014; 44: 382-393.

23 Kalil AC, Metersky ML, Klompas M, et al. Management of Adults With Hospital-acquired and Ventilator-associated Pneumonia: 2016 Clinical Practice Guidelines by the Infectious Diseases Society of America and the American Thoracic Society. Clin Infect Dis 2016; 63: e61-e111.

24 Olivier KN, Griffith DE, Eagle G, et al. Randomized trial of liposomal amikacin for inhalation in nontuberculous mycobacterial lung disease. Am J Respir Crit Care Med 2017; 195: 814-823.

25 Plioplys AV, Kasnicka I. Nebulized tobramycin: prevention of pneumonias in patients with severe cerebral palsy. J Pediatr Rehabil Med 2011; 4: 155-158.

26 Crescimanno G, Marrone O. The microbiome and secondary lung disease in neuromuscular patients: is it time to change our clinical practice? Respirology 2017; 22: 1035-1036.

27 Chatwin M, Tan HL, Bush A, et al. Long term non-invasive ventilation in children: impact on survival and transition to adult care. PLoS One 2015; 10: e0125839.

28 Dohna-Schwake C, Podlewski P, Voit T, et al. Non-invasive ventilation reduces respiratory tract infections in children with neuromuscular disorders. Pediatr Pulmonol 2008; 43: 67-71.

29 Eagle M, Baudouin SV, Chandler C, et al. Survival in Duchenne muscular dystrophy: improvements in life expectancy since 1967 and the impact of home nocturnal ventilation. Neuromuscul Disord 2002; 12: 926-929. 
Sole ML, Talbert S, Penoyer DA, et al. Comparison of respiratory infections before and after percutaneous tracheostomy. Am J Crit Care 2014; 23: e80-e87.

31 Russell CJ, Simon TD, Mamey MR, et al. Pseudomonas aeruginosa and post-tracheotomy bacterial respiratory tract infection readmissions. Pediatr Pulmonol 2017; 52: 1212-1218.

32 Sanders CD, Guimbellot JS, Muhlebach MS, et al. Tracheostomy in children: epidemiology and clinical outcomes. Pediatr Pulmonol 2018; 53: 1269-1275.

33 Stehling F, Steinmann J, Mellies U. The microbiome and secondary lung disease in neuromuscular patients: is it time to change our clinical practice? Reply. Respirology 2017; 22: 1036.

34 Bonfield TL, Panuska JR, Konstan MW, et al. Inflammatory cytokines in cystic fibrosis lungs. Am J Respir Crit Care Med 1995; 152: 2111-2118.

35 Pamukcu A, Bush A, Buchdahl R. Effects of Pseudomonas aeruginosa colonization on lung function and anthropometric variables in children with cystic fibrosis. Pediatr Pulmonol 1995; 19: 10-15.

36 Emerson J, Rosenfeld M, McNamara S, et al. Pseudomonas aeruginosa and other predictors of mortality and morbidity in young children with cystic fibrosis. Pediatr Pulmonol 2002; 34: 91-100.

37 Castellani C, Duff AJA, Bell SC, et al. ECFS best practice guidelines: the 2018 revision. J Cyst Fibros 2018; 17: 153-178.

38 Dhand R. The rationale and evidence for use of inhaled antibiotics to control Pseudomonas aeruginosa infection in non-cystic fibrosis bronchiectasis. J Aerosol Med Pulm Drug Deliv 2018; 31: 121-138.

39 Doring G, Hoiby N. Early intervention and prevention of lung disease in cystic fibrosis: a European consensus. J Cyst Fibros 2004; 3: 67-91.

40 Pai VB, Nahata MC. Efficacy and safety of aerosolized tobramycin in cystic fibrosis. Pediatr Pulmonol 2001; 32 314-327. 